\title{
Use of contrast-enhanced ultrasound for the diagnosis of idiopathic renal hematuria in a dog
}

\author{
Pamela Di Donato ${ }^{1}$, Tiziana Liuti ${ }^{2}$, Jorge Pérez-Accino ${ }^{2}$, Silke Salavati Schmitz ${ }^{2}$, Alexis Trivino $^{2}$ and \\ Maurizio Longo ${ }^{2^{*}}$ \\ ${ }^{1}$ Centro Traumatologico Ortopedico Veterinario, C.Festa 9, 16011 Arenzano (GE), Italy \\ ${ }^{2}$ Royal (Dick) School of Veterinary Studies and Roslin Institute, The University of Edinburgh, Roslin EH259RG, UK
}

\begin{abstract}
Background: Idiopathic renal hematuria (IRH) generally occurs in healthy large-breed dogs that are $<2$-years-old. It is characterized by recurrent bleeding from the kidneys of unidentified cause. The final diagnosis is reached through the exclusion of primary urinary system and systemic causes of hematuria along with the direct visualization by cystoscopy of hematic urine jets at the ureteral orifice.

Case Description: An 8-year-old female neutered Whippet was presented for investigation of a 4-week history of chronic intermittent macroscopic hematuria. Physical examination, systolic blood pressure, extensive laboratory workup (including coagulation profile and platelet count), urine culture, thoracic and abdominal radiographs, pneumocystogram, and double-contrast cystography were all unremarkable. B-mode ultrasound showed no abnormalities apart from a moderate amount of suspended echogenic amorphous material visible within the urinary bladder lumen. In the contrast-enhanced ultrasound (CEUS) study, a large amount of echogenic ill-defined material was noted projecting into the urinary bladder lumen from the right ureterovesical junction in the fundamental mode. This material was more conspicuous and markedly contrast-enhancing in the harmonic mode. Ultrasound contrast medium has the unique property to strictly remain within the vessels without interstitial trapping or elimination by the kidneys. Indeed, the presence of the micro-bubbles into the urinary tract lumen in the CEUS study was interpreted as a direct sign of active urinary tract bleeding. A diagnosis of IRH was reached through CEUS and B-mode ultrasound along with an extensive laboratory workup and periodic follow-up of the patient. No therapy was administered and at a 1-year follow-up, the patient was alive with no current episodes of macroscopic hematuria reported.

Conclusion: To the authors' knowledge, this is the first report describing the use of CEUS for the characterization of IRH. CEUS could represent a safe, non-invasive, affordable novel alternative technique to cystoscopy or cystotomy for the real-time diagnosis of IRH.
\end{abstract}

Keywords: Bleeding, Canine, Kidneys, Ureterovesical jet, Urinary bladder.

\section{Introduction}

Idiopathic renal hematuria (IRH) generally occurs in healthy large-breed dogs less than 2-years-old and without sex predilection (Berent and Weisse, 2013; Berent et al., 2013). This condition is characterized by recurrent bleeding from the kidneys and the cause is undetermined (Kaufmann et al., 1994). The final diagnosis of IRH is reached through the exclusion of causes of primary hematuria from the urinary system or other causes due to systemic illness (thrombocytopenia, inherited or acquired coagulopathy, and hypertension) (Kaufmann et al., 1994). Definitive diagnosis of IRH requires direct visualization of blood tinged urine jets at the ureterovesical junction (UVJ), which is most commonly achieved by cystoscopy, but cystostomy with urethral catheterization has also been described (Gallagher, 2018). To the author's knowledge, no previous report in medicine and veterinary literature has described the use of contrast-enhanced ultrasound (CEUS) for the diagnosis of IRH.

\section{Case Details}

An 8-year-old female neutered Whippet was presented to the Royal (Dick) School of Veterinary Studies (University of Edinburgh) for investigation of a 4-week history of chronic intermittent macroscopic hematuria. The dog had no other lower urinary tract signs and the urine appeared discolored during all the micturition with occasional visible blood clots. The dog received regular flea treatment; vaccination and deworming were up to date. There was no previous history of a bleeding predisposition or trauma. No history of traveling overseas nor history of tick exposure was reported. The dog had been treated by the referring veterinarian surgeon (RVS) with a short course of non-steroidal anti-inflammatories and potentiated amoxycillin with no improvement of the clinical signs. Extensive investigations prior to referral were performed by the RVS and included the following: urinalysis revealed urine specific gravity (USG) of 1.055, $\mathrm{pH} 6$, blood 3+, and protein $3+$; urine sediment revealed a large amount 
of red blood cells. The bacteriologic culture of the urine resulted negative. Hematology and serum biochemistry revealed a mild increase in urea $(8.2 \mathrm{mmol} / 1$, reference $1.7-7.40 \mathrm{mmol} / \mathrm{l})$, mildly increased hemoglobin at $19.7 \mathrm{~g} / \mathrm{dl}(12.00-18.00 \mathrm{~g} / \mathrm{dl})$, and hematocrit at $58.0 \%$ $(37 \%-55 \%)$ suspected to be breed-related. Platelet count was normal. Plain abdominal radiographs of the abdomen, pneumocystogram, and double-contrast cystography had revealed no abnormalities other than an intrapelvic location of the urinary bladder silhouette of equivocal clinical significance. On presentation to our referral center, physical examination was unremarkable, but signs of discomfort were elicited upon palpation of the dorsocaudal abdomen. Differential diagnoses considered for hematuria included a clotting disorder, urinary tract infection or pyelonephritis, neoplasia, trauma, urolithiasis, or IRH. Repeat complete blood count and serum biochemistry were within normal limits. Prothrombin time, activated partial thromboplastin time, D-dimers, and fibrinogen were within the reference intervals. Buccal mucosal bleeding time was less than 4 minutes. Systolic blood pressure was mildly increased $(165 \mathrm{mmHg})$ but not considered high enough to be the cause of bleeding. Repeated urinalysis from both a free catch and a cystocentesis urine sample showed similar findings: macroscopic hematuria, USG of 1.040, $\mathrm{pH} 7$, protein $3+$, and blood 3+. Sediment showed large amounts of erythrocytes with no signs of crystals, casts, or inflammatory cells. Hemoglobinuria or myoglobinuria was excluded by centrifugation. Repeat urinary culture resulted in no bacterial growth. Thoracic radiographs, performed to rule out neoplastic/metastatic changes, were unremarkable. B-mode full abdominal ultrasound and CEUS study of the urinary system were performed using a multifrequency microconvex transducer (CA

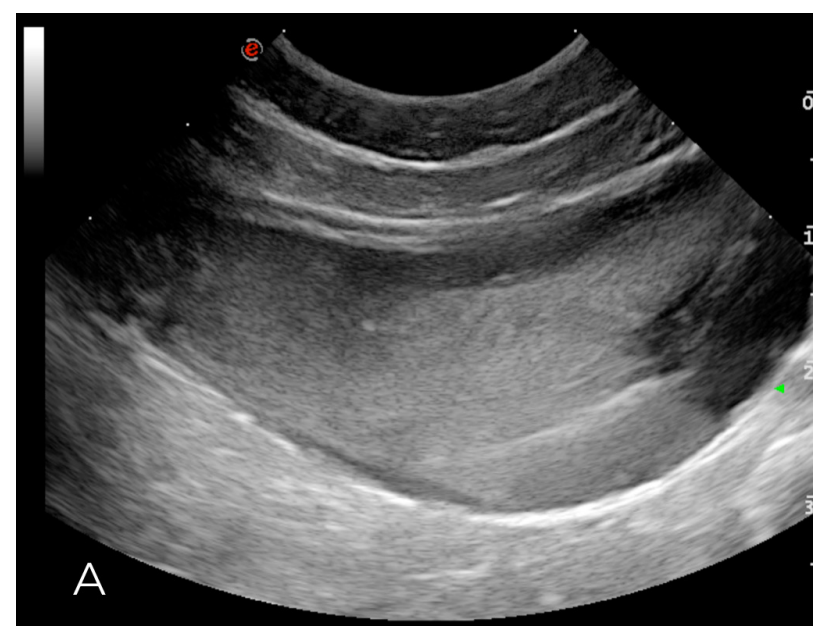

123 Esaote, Genova, Italy). On CEUS, the mechanical index was set at minimum (0.08) and only a single focal zone was positioned, directly below each kidney and urinary bladder. A second-generation contrast agent (SonoVue $C$ Bracco, Milano, Italy) was injected as a bolus into the cephalic vein through an angiocatheter, to evaluate the entire urinary tract. Two different boluses of $0.06 \mathrm{ml} / \mathrm{kg}$ were administered at 10 minutes intervals, followed by injection of a bolus of $3 \mathrm{ml}$ of $0.9 \%$ sodium chloride flush. Images were stored digitally and subsequently analyzed. Moderate amount of suspended echogenic material was visible in the urinary bladder lumen in B-mode (Fig. 1A). During pre-contrast B-mode imaging, the jets from the UVJs were not clearly seen. On CEUS, an intermittent jet of echogenic material from the right-UVJ was noted in the fundamental mode. This finding was more conspicuous with harmonic imaging where intermittent excretion of markedly enhancing mobile material was visible from the right-UVJ (Fig. 1B). No evidence of hyperechoic fountain-like material emerging from the left ureteric orifice was detected. Normal enhancement of the urinary bladder wall was detected with hyperenhancing submucosal layer, serosal layers, and hypoenhancing muscular layer. On B-mode and CEUS ureters, urethra and urinary bladder wall were unremarkable. Kidneys showed a normal architecture, size, and echogenicity, apart for a single left renal cortical cyst $(2 \mathrm{~mm})$ that was considered an incidental finding. Contrast study of both kidneys was also unremarkable with a progressive cortical, corticomedullary, and washout phase. The ultrasonographic findings were indicative of urinary bladder sedimentation most likely consistent with hematuria, likely secondary to active upper urinary tract bleeding from the right nephro-ureteral tract. The most likely differential diagnosis considered was

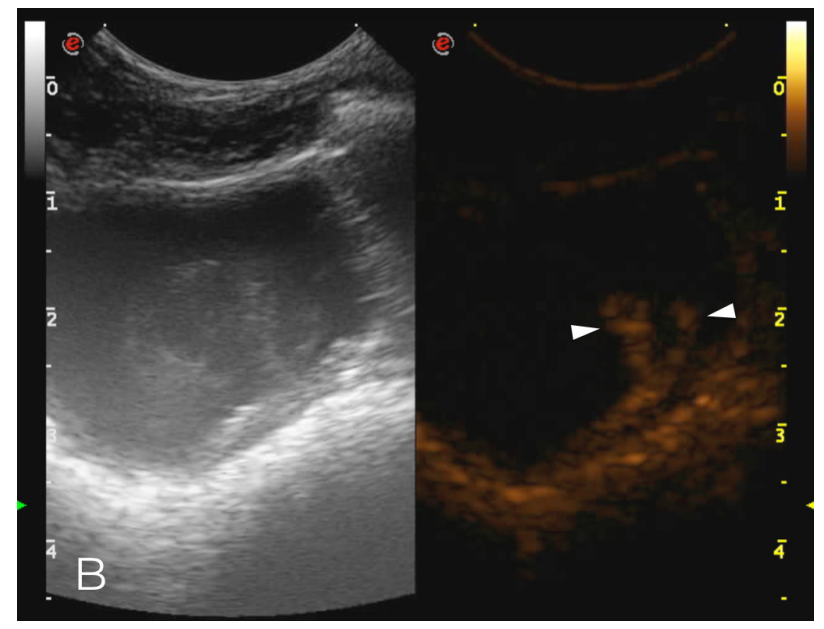

Fig. 1. Long-axis view of the urinary bladder on B-mode (A) and side-by-side B-mode (left) and contrast-mode (right) after administration of contrast agent at the level of the right UVJ (B) A-Notice the large amount of suspended echogenic material occupying most of the lumen of the urinary bladder. B-Notice the large amount of echogenic ill-defined material projecting into the urinary bladder lumen from the right UVJ in fundamental mode (left). This material is markedly contrast-enhancing and more conspicuous with harmonic mode (right; white arrowheads). 
idiopathic renal/ureteral hematuria. Infectious diseases affecting the urogenital tract were excluded due to the negative microbiology exam of the urine. The presence of a neoplastic disease or occult urolithiasis was considered unlikely due to the absence of crystalluria, normal abdominal radiography and normal B-mode, and CEUS studies of the urinary system.

A diagnosis of IRH was made, most likely originating from the right kidney. Cystoscopy with direct visualization of the UVJ, and eventual angio-CT (to rule out subtle vascular lesions of the renal parenchyma or pelvis including arteriovenous malformation, telangiectasias, and aneurysms) were recommended for further assessment but were declined by the owner. Periodical monitoring for signs of anemia and/or signs consistent with lower urinary tract disease (stranguria, dysuria, and urinary obstruction) was recommended. One month later, the dog still had mild intermittent hematuria with no signs of anemia or iron deficiency on complete blood count. At 1-year follow-up, the patient is alive with no current episodes of macroscopic hematuria reported.

\section{Discussion}

IRH typically occurs in healthy large-breed dogs, less than 2-years-old and without sex predilection (Berent and Weisse, 2013; Berent et al., 2013). This condition is characterized by recurrent bleeding from the kidneys where the cause is undetermined (Kaufmann et al., 1994). Hematuria is the only sign in numerous cases, but urinary tract obstruction from blood clots or systemic signs of anemia can also occur (Holt, 1995). In humans, it is defined as acute, intermittent, or chronic gross hematuria for which hematologic, radiologic, and urinalysis (except for the presence of red blood cells) evaluation reveal no clear cause of bleeding (Tawfiek and Bagley, 1998). The final diagnosis of IRH is reached through exclusion of causes of primary hematuria from the urinary system (trauma, inflammatory/infectious, polycystic kidney disease, neoplasia, uroliths, and renal vascular abnormalities) or other causes due to systemic illness (hypertension, thrombocytopenia, inherited, or acquired coagulopathy) (Kaufmann et al., 1994). Definitive diagnosis of IRH requires direct visualization of hematic urine jets at the UVJ, which is most commonly achieved by cystoscopy, but cystostomy with urethral catheterization has also been described (Gallagher, 2018). Generally, if hematuria is the only clinical sign reported as in the presented case, no further therapy is necessary but periodic packed cell volume or hematocrit monitoring is indicated. If the bleeding is severe, medical and surgical treatment, electrocauterization, and/or sclerotherapy/chemical cauterization are necessary (Berent and Weisse, 2013; Berent et al., 2013). Nephrectomy was recommended if the unilateral disease was documented and uncontrollable (Berent and Weisse, 2013; Berent et al., 2013; Holt, 1995; Stones et al., 1983). This is debatable as more than $25 \%$ of the patients can develop bilateral disease and because lesions seem to occur more in the pelvis than within the renal parenchyma (Berent and Weisse, 2013; Berent et al., 2013; Gallagher, 2018). In human medicine, IRH has been described to be correlated with the presence of ruptured hemangiomas, vascular abnormalities with secondary spontaneous bleeding in the renal pelvis (Tawfiek and Bagley, 1998), while in veterinary medicine the cause is not clearly defined (Berent et al., 2013). Similar etiology to humans has been also proposed in the dog (Hawthorne et al., 1998) even if in many cases, histopathology rarely was able to define the cause (Holt, 1995; Stone et al., 1983). In the case described here, extended investigations including systolic blood pressure measurement, coagulation testing and platelets count, urinalysis with microbiology, contrast radiography, and abdominal ultrasound were used to rule out a number of other possible local or systemic causes of hematuria. The present case report is focused on highlighting a novel diagnostic imaging technique for the visualization of the presumed ureteral bleeding, enhancing visualization of the jet by using advanced ultrasound contrast media (CM) and software to enable CEUS of the urinary bladder and UVJ. Being able to visualize blood using CEUS as part of the ureteral jet is less invasive than direct visualization of the UVJ by cystoscopy. In the present case, the large amount of echogenic jet noted in fundamental mode during contrast-enhanced imaging is most likely related to the combination of red blood cells and microbubbles. This finding was even more noticeable using harmonic mode due to the increase conspicuity of the microbubbles (Darge, 2008) combined with the suppression of signal coming from the stationary tissue, e.g., intra-luminal blood clot. Therefore, CEUS harmonic imaging proved to be useful in recognizing the jet even in the presence of a large amount of echogenic material within the urinary bladder lumen. The CM used is made by small $(<7 \mu \mathrm{m})$ microbubbles of inert gas (sulfur hexafluoride) covered by a shell of phospholipids membrane. It remains intravascular and produces a non-linear harmonic response that can be selectively separated from the tissue or fundamental signal using contrast harmonic ultrasound (Darge, 2008). These microbubbles are small enough to be able to pass through the capillaries and be eliminated via the lung but not able to cross the endothelial fenestrations leading to long-time persistence in the bloodstream. Therefore, differently from the $\mathrm{CM}$ used in computed tomography (CT) or magnetic resonance imaging (MRI), which spreads quickly to the extravascular interstitial space, the ultrasound CM has the characteristic to strictly remain confined within the vessel lumen, without interstitial trapping or elimination by the kidneys (Cagini et al., 2013). Indeed, the presence of the micro-bubbles into the urinary tract lumen in the CEUS study should represent a direct sign of active urinary tract bleeding. The use of CEUS to detect intraand extra-parenchymal active bleeding, which is seen as 
extravasation/pooling of microbubbles or hyperechoic bands, has been described in medicine (Miele et al., 2016). This technique, when compared to cystoscopy or even cystotomy, is a safe and non-invasive alternative that does not require general anesthesia, endoscopic equipment, or extensive training. The contrast study is well tolerated even without sedation of the patient. No nephrotoxicity or increased risk of death is related to the CM administration in both dogs and cats (Seiler et al., 2013). The intermittent nature of IRH can hamper its diagnosis. However, this is also a limitation of cystoscopy, which can reveal grossly normal urine jets if there is no active bleeding at the time of the examination, leading to repeated anesthetics. Limitations of this case include the lack of a definitive diagnosis with histopathology and the absence of cystoscopy as a direct comparison of the CEUS findings with the gold standard technique to diagnose IRH. To the authors' knowledge, this is the first report describing the use of CEUS for the characterization of IRH. CEUS could represent a safe, non-invasive, repeatable, affordable novel alternative technique to cystoscopy or cystotomy for the real-time diagnosis of canine IRH. Further prospective studies focused on a larger group of patients, with cystoscopy or surgical confirmation, are necessary to explore the utility of this technique.

\section{Conflict of interest}

The authors declare that there is no conflict of interest. Authors' contribution

Conception and design: Maurizio Longo, Pamela Di Donato, and Tiziana Liuti. Acquisition, analysis, and interpretation of data: Maurizio Longo, Pamela Di Donato, Tiziana Liuti, Jorge Pérez-Accino, Silke Salavati Schmitz, and Alexis Trivino. Drafting the article: Maurizio Longo, Pamela Di Donato, and Jorge Pérez-Accino. Revising article for intellectual content: Maurizio Longo, Pamela Di Donato, Tiziana Liuti, Jorge Pérez-Accino, Silke Salavati Schmitz, and Alexis Trivino. Final approval of the completed article: Maurizio Longo, Pamela Di Donato, Tiziana Liuti, Jorge PérezAccino, Silke Salavati Schmitz, and Alexis Trivino.

\section{References}

Berent, A.C. and Weisse, C. 2013. Idiopathic renal hematuria/benign essential renal hematuria. In Small animal soft tissue surgery, 1st ed. Ed., Monnet E. John Wiley \& Sons, pp: 615-622.
Berent, A.C., Chick, W.W., Branter, E., Adams, L.G., Aarhus, A., Smee, N., Berg, R. and Bagley, D.H. 2013. Endoscopic-guided sclerotherapy for renalsparing treatment of idiopathic renal hematuria in dogs: 6 cases (2010-2012). J. Am. Vet. Med. Assoc. 242(11), 1556-1563.

Cagini, L., Gravante, S., Malaspina, C.M., Cesarano, E., Giganti, M., Rebonato, A., Fonio, P. and Scialpi, M. 2013. Contrast enhanced ultrasound (CEUS) in blunt abdominal trauma. Crit. Ultrasound $\mathrm{J}$. $5(1)$, S9.

Darge, K. 2008. Voiding urosonography with ultrasound contrast agents for the diagnosis of vesicoureteric reflux in children. Pediatr. Radiol. 38, 40-53.

Gallagher, A. 2018. Interventional radiology and interventional endoscopy in treatment of nephroureteral disease in the dog and cat. Vet. Clin. North Am. Small Anim. Pract. 48(5), 843-862.

Hawthorne, J.C., DeHaan, J.J., Goring, R.L., Randall, S.R., Kennedy, F.S., Stone, E. and Zimmerman, K.S. 1998. Recurrent urethral obstruction secondary to idiopathic renal hematuria in a puppy. J. Am. Anim. Hosp. Assoc. 34, 511-514.

Holt, P.E. 1995. Idiopathic renal hemorrhage in the dog. Vet. Rec. 136(2), 52.

Kaufman, A.C., Barsanti, J.A. and Selcer, B.A. 1994. Benign essential hematuria in dogs. Compend. Contin. Educ. Pract. Vet. 16, 1317-1322.

Miele, V., Piccolo, C.L., Galluzzo, M., Ianniello, S., Sessa, B. and Trinci M. 2016. Contrast-enhanced ultrasound (CEUS) in blunt abdominal trauma. Br. J. Radiol. 89, 1-15.

Seiler, G.S., Brown, J.C., Reetz, J.A., Taeymans, O., Bucknoff, M., Rossi, F., Ohlerth, S., Alder, A., Rademacher, N., Drost, W.T., Pollard, R.E., Travetti, O., Pey, P., Saunders, J.H., Shanaman, M.M., Oliveira, C.R., O’Brien, R.T. and Gaschen, L. 2013. Safety of contrast-enhanced ultrasonography in dogs and cats: 488 cases (2002-2011). J. Am. Vet. Med. Assoc. 242, 1255-1259.

Stone, E.A., DeNovo, R.C. and Rawlings, C.A. 1983. Massive hematuria of nontraumatic renal origin in dogs. J. Am. Vet. Med. Assoc. 183, 868-871.

Tawfiek, E.R. and Bagley, D.H. 1998. Ureteroscopic evaluation and treatment of chronic unilateral hematuria. J. Urol. 160, 700-702. 